\title{
Pulsed field gradient magic angle spinning NMR self-diffusion measurements in liquids
}

\author{
Stéphane Viel ${ }^{\mathrm{a}, *}$, Fabio Ziarelli ${ }^{\mathrm{b}}$, Guilhem Pagès ${ }^{\mathrm{a}, 1}$, Caroline Carrara ${ }^{\mathrm{a}}$, Stefano Caldarelli a \\ a Aix-Marseille Université, JE2421 TRACES, av. Escadrille Normandie Niémen, case 512, 13397 Marseille cedex 20, France \\ ${ }^{\mathrm{b}}$ CNRS, Fédération des Sciences Chimiques de Marseille, Spectropole, av. Escadrille Normandie Niémen, case 511, 13397 Marseille cedex 20, France
}

Received 4 September 2007; revised 17 October 2007

Available online 19 November 2007

\begin{abstract}
Several investigations have recently reported the combined use of pulsed field gradient (PFG) with magic angle spinning (MAS) for the analysis of molecular mobility in heterogeneous materials. In contrast, little attention has been devoted so far to delimiting the role of the extra force field induced by sample rotation on the significance and reliability of self-diffusivity measurements. The main purpose of this work is to examine this phenomenon by focusing on pure liquids for which its impact is expected to be largest. Specifically, we show that self-diffusion coefficients can be accurately determined by PFG MAS NMR diffusion measurements in liquids, provided that specific experimental conditions are met. First, the methodology to estimate the gradient uniformity and to properly calibrate its absolute strength is briefly reviewed and applied on a MAS probe equipped with a gradient coil aligned along the rotor spinning axis, the so-called 'magic angle gradient' coil. Second, the influence of MAS on the outcome of PFG MAS diffusion measurements in liquids is investigated for two distinct typical rotors of different active volumes, 12 and $50 \mu \mathrm{L}$. While the latter rotor led to totally unreliable results, especially for low viscosity compounds, the former allowed for the determination of accurate self-diffusion coefficients both for fast and slowly diffusing species. Potential implications of this work are the possibility to measure accurate self-diffusion coefficients of sample-limited mixtures or to avoid radiation damping interferences in NMR diffusion measurements. Overall, the outlined methodology should be of interest to anyone who strives to improve the reliability of MAS diffusion studies, both in homogeneous and heterogeneous media. (C) 2007 Elsevier Inc. All rights reserved.
\end{abstract}

Keywords: HR MAS; Self-diffusion coefficients; Liquids; Gradient uniformity; Magic angle gradient

\section{Introduction}

Diffusion nuclear magnetic resonance (NMR) spectroscopy is nowadays a well established technique for characterizing the structure and dynamics of all kinds of physicochemical systems. Typically, this technique relies on pulsed field gradients (PFG) to label the spatial position of the nuclear spins and infer the molecular diffusion coefficient from their translational displacement over a given time period [1].

\footnotetext{
* Corresponding author. Fax: +33 491288900.

E-mail address: s.viel@univ-cezanne.fr (S. Viel).

${ }^{1}$ Present address: University of Sydney, School of Molecular and Microbial Biosciences, Sydney 2006, Australia.
}

In this context, pulsed gradient spin echo (PGSE) [2] and, more recently, diffusion ordered NMR spectroscopy (DOSY) [3], have become invaluable tools to investigate molecular organization and phase structure [4,5], polymer chain dynamics [6], porous media [7], and to measure thermodynamic binding constants [8] and exchange processes rates [9].

High resolution magic angle spinning (HR MAS) combines high resolution NMR experiments, typically borrowed from liquid state NMR, with magic angle spinning used for reducing both residual dipolar and susceptibility broadenings. In other words, this technique is perfectly adapted to the study of viscous or gel-like samples, the so-called semi-solid class of samples, and has generated a large variety of applications $[10,11]$. 
Further improvement in HR MAS studies has been achieved with the introduction of gradient enhanced NMR spectroscopy after the successful implementation by Maas et al. [12] of a gradient coil in a HR MAS probe. In fact, because sample spinning introduces additional modulations (e.g. $B_{1}$ and $Q$ modulations), suppression of $t_{1}$ noise in two-dimensional correlation experiments is even more important in HR MAS than in the liquid state. In the implementation proposed by Maas et al. [12], the magnetic field gradient coil is aligned along the spinner axis, in order to avoid rotational averaging of the magnetic field gradient and ensure that a rotating, non-diffusing spin packet always experiences the same gradient strength. Because the spinner axis is by definition oriented along the magic angle in HR MAS, this specific gradient coil configuration is usually referred to as 'magic angle gradient'. Note in passing that the 'magic angle gradient' is a static field gradient. Alternatively, radiofrequency field $B_{1}$ gradients can also be used, as recently evidenced by Malveau et al. [13].

In addition to improving the spectral quality in multidimensional MAS experiments [14], the introduction of a magic gradient coil in HR MAS probes has allowed PFG experiments to be efficiently performed on semi-solid samples. Many applications have been reported, from the design of novel diffusion-edited pulses sequences $[15,16]$, to the investigation of diffusional processes in semi-solid samples, such as biological tissues [17], human cells [1820], liposomes [21], membranes [22,23], liquid crystals [24], and foods [25].

In the mean time, this gradient technology was also adapted to solid state MAS probes and used for selecting coherence pathways or suppressing unwanted NMR signals in solid state MAS experiments [26,27]. However, investigations of diffusional processes in the solid state have been rather limited so far, mainly because standard MAS probes do not afford sufficiently high gradient strengths to achieve meaningful results in PFG studies of slowly diffusing species. To overcome this difficulty, Pampel et al. have advantageously combined a MAS probe with a microimaging gradient system that offers, with respect to conventional MAS probes, a 10 -fold increase in gradient strength [28]. Applications of this new technical development seem promising, especially for the investigation of multi-component diffusion in zeolites [29].

Interestingly, while diffusion coefficients are now routinely measured in MAS studies of heterogeneous media, at least for semi-solid samples, the possibility to measure by PFG MAS the self-diffusion coefficients of pure liquids and solutions has been scarcely addressed in the literature so far. To the best of our knowledge, the sole published report is the recent study by Bradley et al. [30]. In particular, these authors investigated the possibility to record DOSY spectra on sample-limited mixtures by using three distinct types of probes (e.g. conventional, cold, and nano probes). They showed that, in their experimental setup, the nano probe performed worse than the cold one. This was explained by the presence in the nano probe of an additional flow due to vortexing caused by sample spinning, which led to erratic signal intensities. Because these errors could be suppressed somehow by extensive signal averaging, a rather typical practice for sample-limited studies, their conclusion was that DOSY maps of acceptable quality could be obtained for these systems as long as purely qualitative results were sought.

Still, the possibility to achieve quantitative diffusion coefficients in PFG MAS experiments is of interest for at least two reasons. First, it remains so far the only available opportunity for investigating the diffusional behaviour of samples present in very little amount, although a promising and totally original alternative has been very recently evoked by Maguire et al. [31]. Second, because MAS can efficiently remove dipolar field effects observed in liquids [32], MAS diffusion studies offer a nice option to avoid complications caused by radiation damping in NMR diffusion measurements of pure liquids or concentrated mixtures on modern high field instruments $[33,34]$.

In this work, we emphasize the experimental conditions required to achieve accurate measurements of self-diffusion coefficients in PFG MAS diffusion studies of liquids. Overall, we believe that the outlined methodology will be of interest to anyone who strives to improve the reliability of PFG MAS diffusion studies both in homogeneous and heterogeneous media. As a matter of fact, this specific investigation was originally initiated in the framework of our ongoing research project on MAS diffusion studies in heterogeneous media involving chromatographic stationary phases [35-37], within which we have been inquiring on the accuracy and precision of self-diffusion measurements performed by PFG MAS.

Specifically, after listing all relevant experimental details in Section 2, we review and apply in Section 3 the required methodology to check the uniformity and calibrate the strength of the gradient coil. Finally, Section 4 illustrates the influence of MAS on the outcome of PFG MAS diffusion measurements performed in liquids, for two distinct standard rotors of different active volumes (12 and $50 \mu \mathrm{L}$ ). The results show that, with appropriate experimental settings, accurate self-diffusion coefficients can be obtained by PFG MAS experiments, both for fast and slowly diffusing species.

\section{Experimental}

\subsection{Samples and materials}

For the gradient calibrations, two samples were used: a home-made $\mathrm{H}_{2} \mathrm{O} / \mathrm{D}_{2} \mathrm{O}(50 / 50$, v/v) sample doped with $\mathrm{CuSO}_{4}$, and a $99.9 \% \mathrm{D}_{2} \mathrm{O}$ sample. Except for water, which was purified by using a microporous filtration system (Millipore S.A.), all non deuterated solvents used in this study were high purity solvents (HPLC grade) purchased from Sigma-Aldrich, whereas the deuterated solvents were from Eurisotop. The poly(ethylene oxide) sample $\left(M_{\mathrm{w}} 116 \mathrm{kDa}\right)$ 
was purchased from Sigma-Aldrich. All chemicals were used as received.

All experiments were recorded using $4 \mathrm{~mm}$ o. d. Zirconia rotors sealed with Kel-f caps and equipped (or not) with PTFE inserts (CortecNet). These rotors allowed for complete air removal during sample preparation. Three distinct rotors were used: a $100 \mu \mathrm{L}$ rotor for the gradient profile experiment, and a 12 and $50 \mu \mathrm{L}$ rotors for diffusion measurements. Finally, a rotor prototype [38] was used to calibrate the absolute value of the gradient strength.

\section{2. $N M R$}

All NMR experiments were performed on a BRUKER Avance 400 spectrometer operating at $400.13 \mathrm{MHz}$ for the ${ }^{1} \mathrm{H}$ Larmor frequency with a BRUKER $4 \mathrm{~mm}{ }^{1} \mathrm{H} /{ }^{13} \mathrm{C}$ HR MAS probe equipped with a ${ }^{2} \mathrm{H}$ lock channel and a magic angle gradient coil. Temperature control was ensured by controlling the bearing gas temperature with a BCU05 and BVT3300 unit from BRUKER.

NMR diffusion measurements were performed using the pulse sequence proposed by $\mathrm{Wu}$ et al. [39], based on the stimulated echo, which incorporates bipolar gradient pulses and a Longitudinal Eddy current Delay (LED). Here, two negative spoiler gradients $(2 \mathrm{~ms}$ duration at about $20 \%$ of the maximum gradient strength) were also applied during the $z$ storage periods. In all experiments, sine gradient pulses were used and the LED was held constant at $5 \mathrm{~ms}$.

Typically, all delays were kept constant to avoid complication arising from magnetic relaxation, and only the gradient strength was varied. Specifically, the gradient strength was quadratically incremented in 16 steps from $6 \%$ to $95 \%$ of its maximum value, and 16 transients were accumulated for each gradient value. For a given diffusion time, the gradient pulse durations were optimized to achieve at the largest gradient amplitude a decrease in the resonance intensity higher than $95 \%$. Although the diffusion experiments performed on the non deuterated solvents were recorded without lock, this was not a problem considering the relatively short duration of the experiments ( 25 min).

After Fourier transformation and phase correction, the baseline of the spectra was carefully adjusted. The data were analyzed as reported in [4] by plotting the signal intensities (areas) as a function of the gradient strength and fitting the resulting PFG decays to the appropriate StejskalTanner equation [39] with a nonlinear least-squares fit based on the standard Levenberg-Marquardt algorithm (implemented on the Sigmaplot 8.0 software from SPSS, Inc.). Only single exponential fits were performed.

\section{Temperature and gradient coil calibrations}

To achieve accurate diffusion measurements, a whole series of preliminary calibrations is required. In this context, extensive literature is available and, for recent reviews, the interested reader is referred for instance to references [40-43]. The next three sections apply some of the concepts outlined in these works, focusing especially on the specific application of PFG MAS studies.

\subsection{Temperature calibration}

As a prerequisite, any diffusion study requires proper control and assessment of sample temperature. Temperature calibration of a HR MAS probe within the 280 $310 \mathrm{~K}$ range has already been reported in the literature [44], and these authors showed that, for a given temperature setting, increasing the spinning rate from 2 to $8 \mathrm{kHz}$ led to an increase in sample temperature of about $8 \mathrm{~K}$. Additional heating may occur in samples with high electrical conductivity. The practical implication of this for diffusion measurements is that temperature should be assessed as a function of the spinning rate. Accordingly, although the working temperature was always set to $298 \mathrm{~K}$ in this study (unless otherwise stated), the actual sample temperature was systematically verified by analyzing neat methanol, used as a NMR thermometer [45], under the same experimental conditions (rotor type and spinning rate).

Interestingly, note that the issue of possible temperature gradients at the sample in HR MAS has been rather overlooked in the literature so far [30,46], although this specific matter should be of relevance in the investigation of heterogeneous systems and semi-solid samples. In fact, temperature gradients are known to form in solid state MAS NMR [47]. However, in the present study, this issue can be safely ignored mainly because all measurements were performed at room temperature on rather small active volume samples with spinning rates higher than $2 \mathrm{kHz}$ that imply the use of large bearing gas flows (more than $30 \mathrm{~L} \mathrm{~min}^{-1}$ ). All together, these combined features preclude the formation of significant temperature gradients.

\subsection{Gradient coil uniformity}

To evaluate the gradient uniformity [48], we used the pulse sequence proposed by Hurd et al. [49]. It consists of a simple Hahn echo in which the central 180 pulse is flanked by two gradient pulses $(g)$ and the echo is acquired in the presence of another gradient $\left(g_{\mathrm{a}}\right)$. This sequence was applied to a $100 \mu \mathrm{L}$ rotor containing a $\mathrm{H}_{2} \mathrm{O} / \mathrm{D}_{2} \mathrm{O}(50 / 50$, v/v) sample doped with $\mathrm{CuSO}_{4}$ to generate a magnetization profile over the length of the rotor. This rotor was chosen to sample the whole length of the coil. At this stage, no sample spinning was applied. Results are shown in Fig. 1a.

With weak gradients surrounding the 180 refocusing pulse, the profile (straight line) is a crude measure of $B_{1}$ homogeneity along the length of the coil [38]. However, with a strong gradient pair, the profile (dotted line) becomes heavily diffusion weighted.

By assuming constant temperature and unrestricted diffusion, which is not unreasonable for the analysis of a non spinning liquid, the spatial dependence of the gradient 

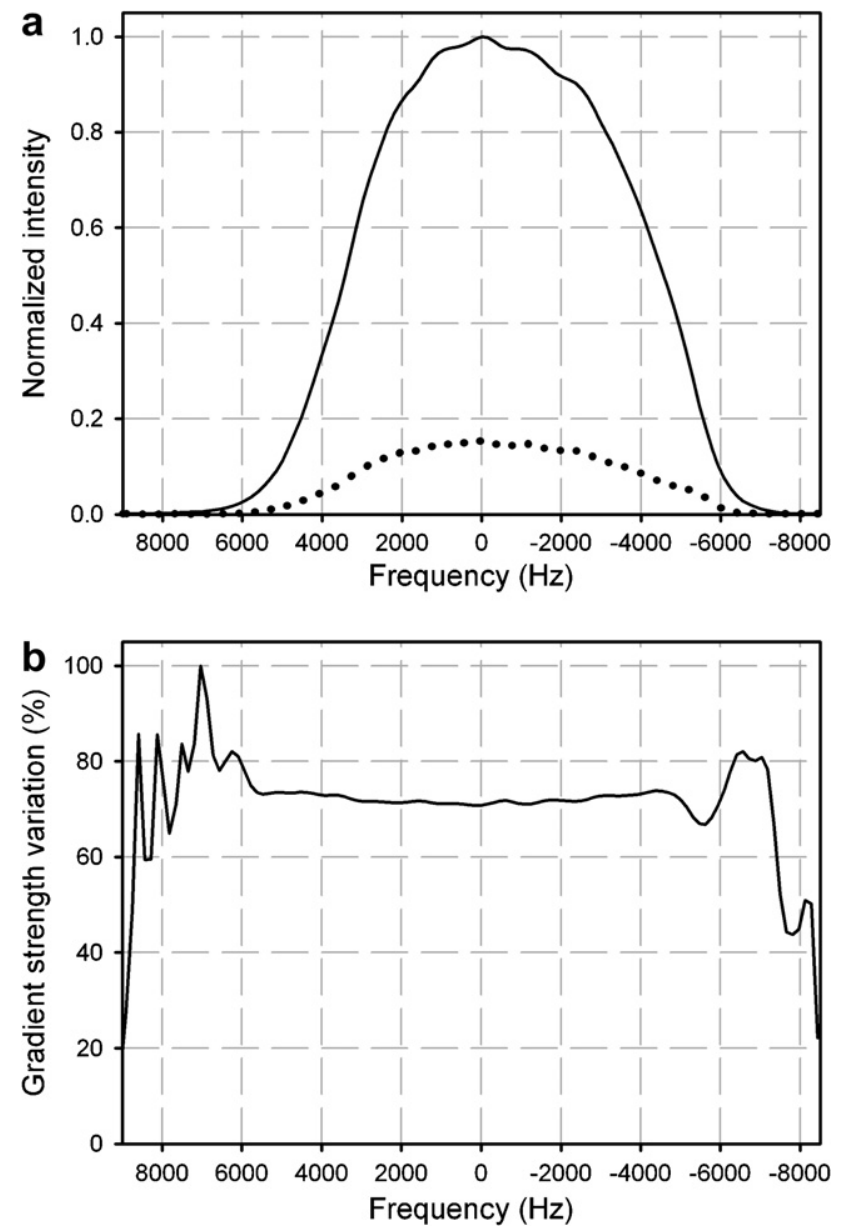

Fig. 1. (a) Images of a non spinning $100 \mu \mathrm{L}$ rotor filled with $\mathrm{H}_{2} \mathrm{O} / \mathrm{D}_{2} \mathrm{O}$ $(50 / 50, \mathrm{v} / \mathrm{v})$ sample doped with $\mathrm{CuSO}_{4}$ obtained on a HR MAS probe equipped with a magic angle gradient with the pulse sequence described by Hurd et al. [49] for two distinct values of the maximum gradient strength $g$, namely $6 \%$ and $50 \%$ for the straight and dotted lines, respectively. In both cases, the diffusion delay $\Delta$ and the gradient pulse duration $\delta$ were set to 40 and $2 \mathrm{~ms}$, respectively, and the acquisition gradient $g_{\text {a }}$ was $5 \%$ of the maximum gradient strength. The frequency spectrum is arbitrarily referenced by setting the frequency of the image point characterized by the highest intensity to zero. (b) Corresponding $z_{\text {MAS }}$ profile of the magic angle gradient calculated according to Eq. (1).

strength along the magic angle axis, the so-called $z_{\text {MAs }}$ gradient profile, can be determined by rewriting the StejskalTanner equation according to

$g=\frac{1}{\gamma \delta \sqrt{D-\left(\Delta-\frac{\delta}{3}\right)}} \sqrt{\ln \left(\frac{I}{I_{0}}\right)}$

where $\gamma$ is the magnetogyric ratio, $\delta$ is the gradient pulse duration, $\Delta$ is the diffusion time, $I_{0}$ and $I$ are the intensity of the NMR signal at low and high gradient strength, respectively, and $D$ is the diffusion coefficient of the analyzed molecular species. Basically, all experimental parameters are known except for $D$. However, because it simply enters as a scale factor, its true value is unimportant for the determination of the gradient profile. The only important point is that it does not vary along the rotor axis, which is unexpected as long as the temperature is uniform. Fig. $1 \mathrm{~b}$ shows the corresponding $z_{\text {MAs }}$ profile. This profile is relatively flat within the $\pm 6 \mathrm{kHz}$ region but presents otherwise large distortions. These are merely due to experimental artefacts, as will be evidenced in the following section.

\subsection{Gradient coil strength}

Two main methods are available to calibrate the gradient strength, which can be defined as absolute or relative. We successively present the results obtained by both methods.

\subsubsection{Absolute gradient calibration}

First, we used the conventional method for measuring the absolute value of the gradient coil constant by recording the image of a phantom of precisely known thickness. To do so, we used a recently introduced rotor design which allowed us to modify the volume of the rotor by placing inside discs of $1 \mathrm{~mm}$ thickness, hereafter referred to as spacers [38]. Specifically, we recorded the image of this rotor filled with the previous $\mathrm{H}_{2} \mathrm{O} / \mathrm{D}_{2} \mathrm{O}$ sample by using successively 3,4 , and 5 spacers. This allowed us to be in the middle of the sample volume of the rotor, where the $B_{1}$ field and the gradient are most homogeneous. Results are shown in Fig. 2a. From these spectra, the absolute value of the gradient coil constant could be calculated according to

$\frac{\Delta v}{\varepsilon}=\frac{\gamma}{2 \pi} \cdot g_{\mathrm{a}} \cdot I_{\mathrm{MAX}} \cdot G_{\mathrm{coil}}$

where $\Delta v$ represents the width $(\mathrm{Hz})$ of the phantom image of thickness $\varepsilon(\mathrm{cm})$ recorded in the presence of a magnetic field gradient of strength $g_{\mathrm{a}}$ (expressed in \% of the maximum gradient strength), $\gamma$ is the ${ }^{1} \mathrm{H}$ magnetogyric ratio $\left(26.751 \times 10^{3} \mathrm{rad} \mathrm{Hz} \mathrm{G}^{-1}\right), I_{\mathrm{MAX}}$ is the maximum current intensity (A) that the gradient amplifier can deliver, and $G_{\text {coil }}$ is the gradient coil constant $\left(\mathrm{G} \mathrm{cm}^{-1} \mathrm{~A}^{-1}\right)$.

In our case, with a $10 \mathrm{~A}$ gradient pulse amplifier, a 5\% acquisition gradient strength $\left(g_{\mathrm{a}}\right)$ experimentally yielded a frequency width $(\Delta v)$ of $1290 \pm 10 \mathrm{~Hz}$, which in turn gave us a gradient coil constant of

$G_{\text {coil }}=6.05 \pm 0.05 \mathrm{G} \mathrm{cm}^{-1} \mathrm{~A}^{-1}$

Finally, by combining all these data, the profile of the gradient coil constant as a function of the position inside the rotor could be obtained, as shown in Fig. 2b. In establishing this profile, only the regions corresponding to a $B_{1}$ field greater than $4 \%$ of its maximum value were considered to avoid artifacts due to the non linear response of the profiles at the rotor edges [49].

As can be seen in Fig. 2b, the gradient is almost perfectly uniform, both when using a 12 and $50 \mu$ L rotor volumes. In other words, recording diffusion experiments on HR MAS probes avoids experimental artifacts caused by gradient poor uniformity (typically evidenced by non linear PFG 

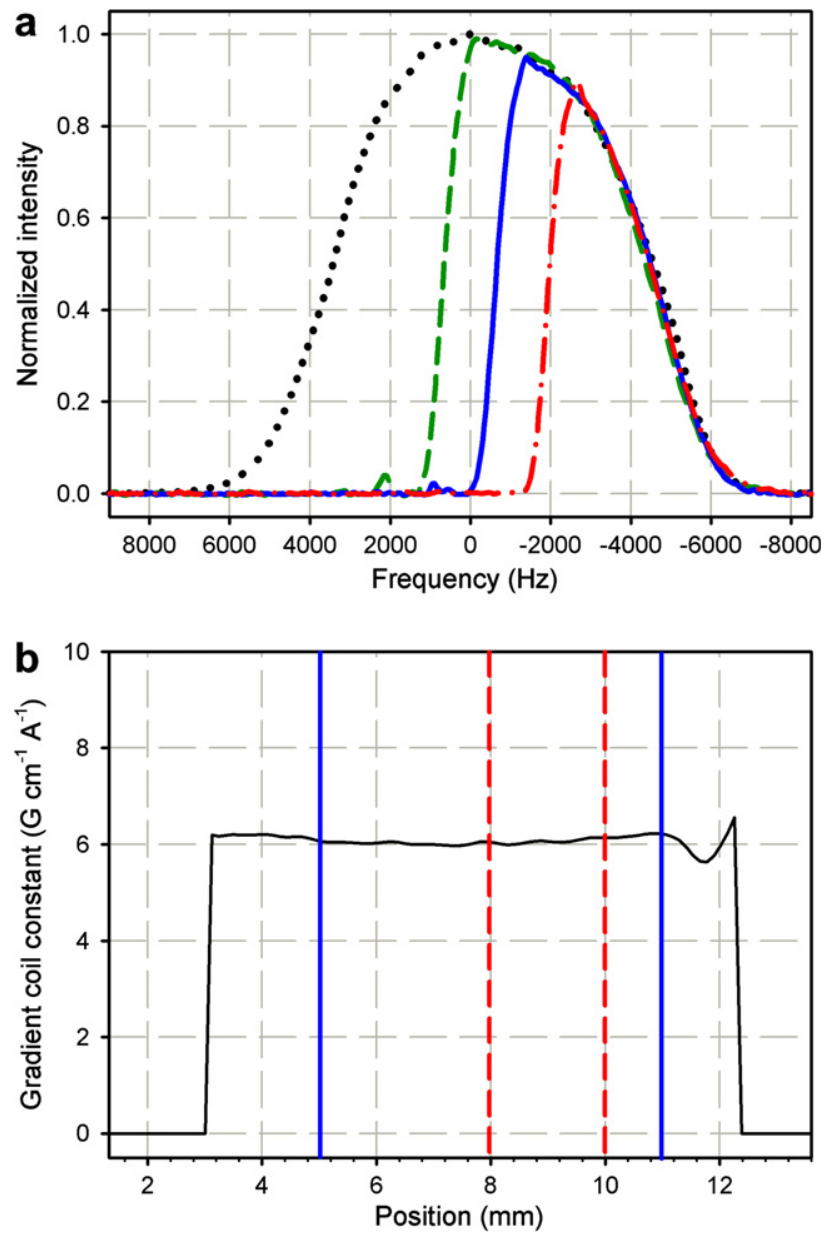

Fig. 2. (a) Images of a non spinning $\mathrm{H}_{2} \mathrm{O} / \mathrm{D}_{2} \mathrm{O}(50 / 50$, v/v) sample doped with $\mathrm{CuSO}_{4}$ obtained as in Fig. 1 with an acquisition gradient strength $g_{\mathrm{a}}$ of $5 \%$ for a $100 \mu \mathrm{L}$ non spinning rotor prototype containing 3 (dashed line), 4 (straight line), and 5 (dot-dashed line) slices of $1 \mathrm{~mm}$ thickness (spacers). For comparison the full rotor profile is also reported (dotted line). The frequency axis is referenced as in Fig. 1. (b) Variation of the magic gradient coil constant as a function of the rotor position. Intensities were truncated at $4 \%$ of the $B_{1}$ field to avoid spurious artifacts due to the non-linearity of the profiles at the edges. As an indication, the sample volumes corresponding to the $12 \mu \mathrm{L}$ (dashed line) and $50 \mu \mathrm{L}$ (straight line) rotor volumes are indicated.

decays), and hence represents an interesting alternative to other setups designed to avoid these artifacts such as, for instance, post processing corrections [48], Shigemi tubes [50], or slice selective experiments [51].

\subsubsection{Relative gradient calibration}

The absolute gradient calibration method is very useful when nothing is known about the strength of the gradient coil. However, its precision critically relies on the precision of the phantom thickness, a point that has been purposely ignored in the previous section as a first approximation. In fact, a change in disc thickness of $\pm 0.01 \mathrm{~cm}$ would imply a change in the gradient coil constant of $\pm 0.6 \mathrm{G} \mathrm{cm}^{-1} \mathrm{~A}^{-1}$, which would roughly lead, for the same signal decay, to a $\pm 20 \%$ change in the diffusion coefficient value. Here, how- ever, such problem is not expected because of the high precision of the discs used.

In any case, a typical method for checking the accuracy of the previously established calibration consists of measuring the diffusion coefficient of a substance of known diffusivity. Conventionally used references are distilled water or $99.9 \%$ deuterated water [40]. Accordingly, both references were analyzed here and two distinct rotors were used: 12 and $50 \mu \mathrm{L}$. For each measurement, two distinct $\Delta$ values were chosen (20 and $80 \mathrm{~ms}$ ), and the durations of the gradient pulses $(\delta / 2)$ were optimized in order to obtain in both cases roughly the same residual intensity at the maximum gradient strength (lower than 5\%). All the results are reported in Table 1 . These were achieved by using the value of the gradient coil constant $\left(G_{\text {coil }}\right)$ calibrated in the previous section.

Importantly, in all cases, no sample spinning was applied and both the bearing and drive gases were turned off. In other words, the sample temperature could not be actively controlled and was actually regulated by the air conditioning system of the laboratory room [52]. The absence of active temperature control for NMR diffusion measurements performed at room temperature has already been reported before [53]. As a matter of fact, for room temperature measurements, it represents an efficient option for avoiding temperature gradients caused by the use of typical variable temperature (VT) units for liquid state NMR experiments recorded on standard $5 \mathrm{~mm}$ tubes [54]. However, in this specific experimental configuration, our temperature control was far from optimal, as will be evidenced later.

Table 1 shows that, by using the absolute value of the gradient coil constant given by Eq. (3), accurate $D$ measurements were obtained for both references. Overall, average values of $2.30 \pm 0.04$ and $1.92 \pm 0.03\left(10^{-9} \mathrm{~m}^{2} \mathrm{~s}^{-1}\right)$ were obtained for $\mathrm{H}_{2} \mathrm{O}$ and $\mathrm{HOD}$, respectively, which agreed well with literature data (2.30 and 1.90, from reference [55]). This implies that the calibration reported in the previous section is accurate, especially when considering the precision of our temperature control $( \pm 2 \%)$ in this particular experimental setup (i.e., without sample spinning).

Table 1

Self-diffusion coefficients measured at $298 \mathrm{~K}$ for a distilled water sample ${ }^{\mathrm{a}}$ and a $99.9 \% \mathrm{D}_{2} \mathrm{O}$ sample ${ }^{\mathrm{b}}$ on a HR MAS probe without sample spinning for a 12 and $50 \mu \mathrm{L}$ rotor

\begin{tabular}{lllll}
\hline Reference & Rotor $(\mu \mathrm{L})$ & $\Delta(\mathrm{ms})$ & $\delta / 2(\mathrm{~ms})$ & $D_{\text {all }}\left(\times 10^{-9} \mathrm{~m}^{2} \mathrm{~s}^{-1}\right)$ \\
\hline $\mathrm{HOH}^{\mathrm{a}}$ & 12 & 20 & 1.6 & $2.30 \pm 0.04$ \\
& & 80 & 0.8 & $2.30 \pm 0.04$ \\
& 50 & 20 & 1.6 & $2.32 \pm 0.04$ \\
$\mathrm{HOD}^{\mathrm{b}}$ & \multirow{2}{*}{12} & 80 & 0.8 & $2.30 \pm 0.04$ \\
& & 20 & 1.6 & $1.93 \pm 0.03$ \\
& 50 & 80 & 0.8 & $1.95 \pm 0.03$ \\
& & 20 & 1.6 & $1.94 \pm 0.03$ \\
& & 80 & 0.8 & $1.90 \pm 0.03$
\end{tabular}

$D_{\text {all }}$ was estimated using the $G_{\text {coil }}$ value given in Eq. (3). 
Furthermore, according to Antalek [41], the diffusion coefficients were also evaluated by only considering the first five points $\left(D_{\text {first five }}\right)$ and the last five points ( $\left.D_{\text {last five }}\right)$ of the corresponding PFG decays, and the values were compared with those obtained by analyzing the full decay $\left(D_{\text {all }}\right)$. This is especially convenient to detect curvature in PFG decays caused by non ideal gradient uniformity. While no difference was observed between $D_{\text {all }}$ and $D_{\text {first five, }} D_{\text {last five was systematically found to be about }}$ $4 \%$ larger than the average $D_{\text {all }}$ value. Considering the high gradient coil uniformity evidenced in Fig. 2b, this deviation is most probably due to sample heating caused by the gradient pulses. In fact, without active temperature control, the last and strongest gradient pulses may slightly heat the sample, and temperature gradients may appear. These are usually detected by a downward curvature in the PFG signal decay at highest gradient strength [41], which is what we actually observed for the experiments performed in these conditions on the non spinning samples.

Finally, another use of the relative calibration method is to account for the eddy current field [56]. Here the values obtained by the absolute and relative methods were very close, which implies that, under these experimental conditions, eddy currents were negligible, which is rather typical for bipolar gradient pulses of limited strength.

\section{Results and discussion}

\subsection{Influence of MAS on diffusion measurements}

To address the influence of MAS on the outcome of PFG MAS diffusion measurements performed in liquids, we first analyzed the diffusion coefficient of two relatively fast diffusing species exhibiting different viscosities, namely water and acetonitrile (1.00 and $0.37 \mathrm{cP}$ at $293 \mathrm{~K})$, as a function of the spinning rate (from 2 to $8 \mathrm{kHz}$ ), both for a 12 and $50 \mu \mathrm{L}$ rotor volume. This range of spinning rates was selected because it corresponded to typically employed values and allowed, on our system, for stable sample rotations $( \pm 3 \mathrm{~Hz})$.

In all cases, although the temperature was set to $25^{\circ} \mathrm{C}$, the true sample temperature was verified for each spinning rate and each rotor [44]. Because the temperature dependence of the self-diffusion coefficients of these two compounds is known [57,58], their measured $D$ values were then corrected in accordance with the true sample temperature. The results are reported in Fig. 3. Specifically, this figure shows the evolution of the ratio of the measured self-diffusion coefficient $\left(D_{\text {meas }}\right)$ to the self-diffusion coefficient value expected at this temperature $(D(T))$, as a function of the spinning rate. Clearly, very different trends are observed for the 12 and $50 \mu \mathrm{L}$ rotors.

For $12 \mu \mathrm{L}$, the spinning rate did not influence the outcome of the PFG measurements performed on water (in agreement with literature data [21]) but slightly modified the results obtained on acetonitrile, especially for the 2 and $8 \mathrm{kHz}$ spinning rates for which very large deviations were observed. Overall, optimal results (deviations lower than $\pm 2 \%$ ) could be achieved for both substances as long as the spinning rate was included between 3 and $6 \mathrm{kHz}$. In other words, for the $12 \mu \mathrm{L}$ rotor, the change in the self-diffusion coefficient value was mostly in agreement with the temperature increase caused by sample spinning.

In contrast, for the $50 \mu \mathrm{L}$ rotor, all measurements were either simply overestimated or totally unreliable. For water, the observed deviation increased with the spinning rate whereas, for acetonitrile, totally unreliable results were obtained. Interestingly, the distinct behaviours observed for water and acetonitrile for both rotors suggested that the physicochemical properties of the analyzed samples may play a role.

Moreover, comparison of the PFG decays obtained for both solvents with both rotors showed that, in contrast to the $12 \mu \mathrm{L}$ rotor, for which satisfactory linear decay curves were obtained, the decays obtained with the $50 \mu \mathrm{L}$ rotor were clearly not linear, as illustrated in Fig. 4 for acetonitrile at $4 \mathrm{kHz}$ in a 12 and $50 \mu \mathrm{L}$ rotor. Because pure monoexponential decays were obviously expected in this case, the observation of an additional faster decaying component for the $50 \mu \mathrm{L}$ rotor indicated the presence of experimental artefacts, which typically lead to enhanced apparent diffusion rates due to excessive and artificial echo attenuation [59], although the opposite trend may also be observed [53]. Thus, because all decays obtained in this work were processed with single exponential fits for consistency, these experimental interferences explained why the corresponding $D$ measurements in the $50 \mu \mathrm{L}$ rotor were systematically overestimated.

As already mentioned, when performing DOSY experiments in liquids on a nano probe at $2.2 \mathrm{kHz}$ with a slightly lower active volume $(40 \mu \mathrm{L})$, Bradley et al. [30] observed the presence of a flow, which they attributed to sample turbulences inside the rotor caused by vortexing due to sample spinning. Clearly, considering the range of spinning rates used in this study, these turbulences are most likely to appear easily in large volume rotors and for low viscosity samples. This may explain why, for low spinning rates, $D$ measurements performed with a $50 \mu \mathrm{L}$ rotor in water are somehow more reliable than those achieved in acetonitrile, the former being more viscous than the latter.

Another possible explanation for these interferences could be that sample spinning causes in some cases mechanical vibrations [60] that perturb the diffusion measurements. Also, note that the geometries of the rotors used in this study were slightly different, the 12 and $50 \mu \mathrm{L}$ active volumes being spherical and (rather) cylindrical, respectively. In other words, to better ascertain this point, further measurements are required, based in particular on the use of different types of rotors (and inserts) [60] as well as solvents exhibiting distinct physical properties (viscosity, density, etc.).

Therefore, in order to achieve accurate diffusion measurements, a small active volume rotor (here $12 \mu \mathrm{L}$ ) and 

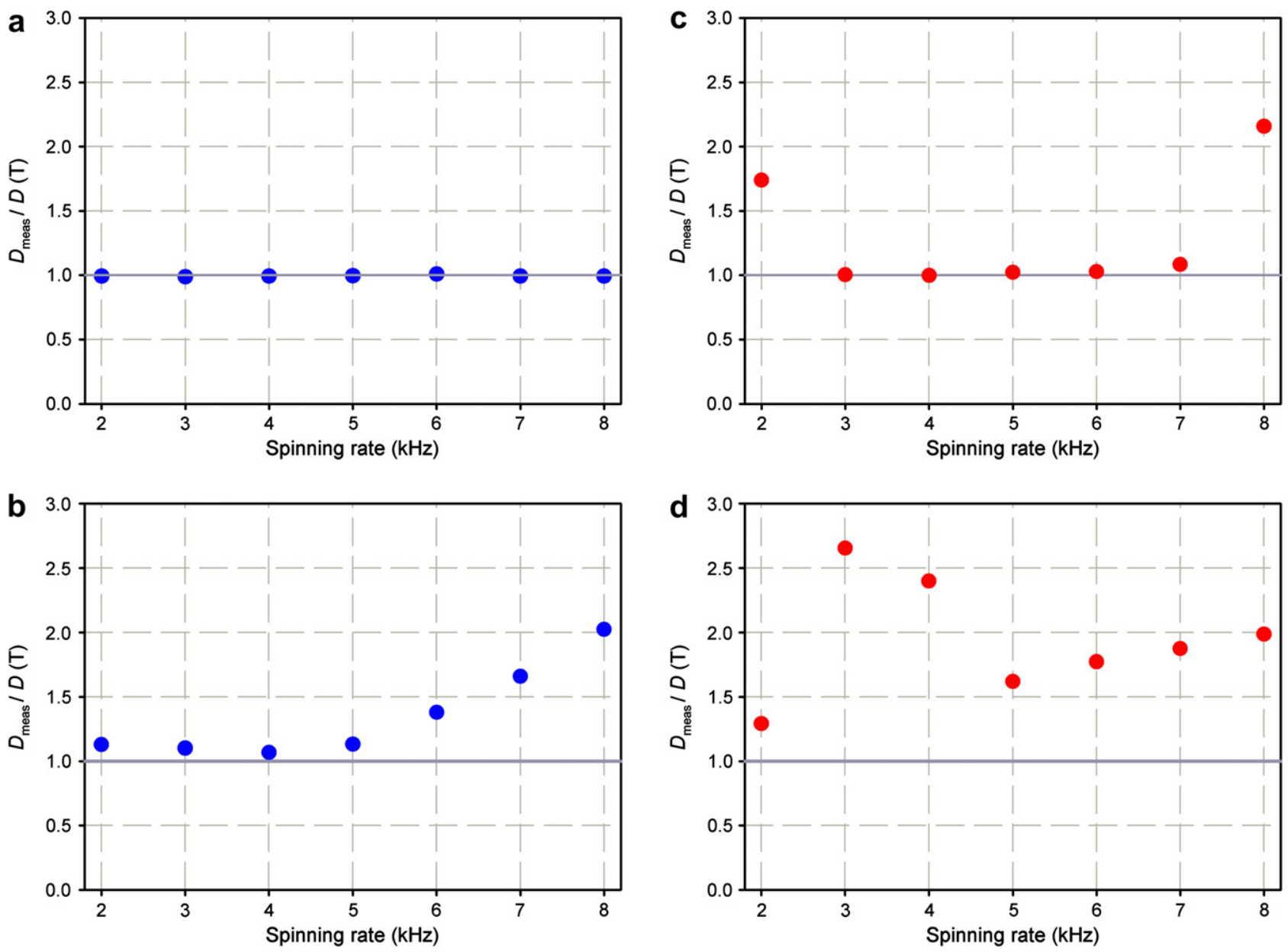

Fig. 3. Evolution as a function of the spinning rate of the ratio of the self-diffusion coefficient value measured by PFG MAS ( $D_{\text {meas }}$ ) to the self-diffusion coefficient value expected at the corresponding temperature $(D(T))$ for distilled water (a and b) and acetonitrile (c and d), obtained for a $12 \mu \mathrm{L}$ rotor (a and c) and a $50 \mu \mathrm{L}$ rotor (b and d). In all cases the diffusion time $\Delta$ was $80 \mathrm{~ms}$ whereas the gradient pulse duration $\delta / 2$ were 0.8 and $0.6 \mathrm{~ms}$ for water and acetonitrile, respectively.

a properly selected spinning rate should be used. This implies that the relative method for calibrating the gradient strength, based on the measurement of the $D$ coefficient for a substance of known diffusivity, should be cautiously employed on a MAS probe in case of sample spinning, since wrong $D$ measurements will clearly lead to wrong estimates of the gradient coil constant. These conclusions obviously apply for measurements performed in liquids. Indeed, when analyzing heterogeneous media, such as semi-solid samples or mixtures of liquid and solid samples, these previously mentioned interferences are most probably absent.

\subsection{PFG MAS diffusion measurements}

At this point, by using the experimental conditions described in the previous section, we determined the selfdiffusion coefficients both of fast and slowly diffusing species.

On one hand, as fast diffusing components, we analyzed by PFG MAS a set of standard solvents exhibiting different viscosities, and for which recent high quality NMR self-diffusion data has been published in the literature [50].

According to Fig. 3, we worked with a $12 \mu \mathrm{L}$ rotor, using a spinning rate of $4 \mathrm{kHz}$ that is usually sufficient to avoid the presence of perturbing spinning side bands in the $400 \mathrm{MHz}{ }^{1} \mathrm{H}$ spectrum. The diffusion time $\Delta$ was systematically set to $80 \mathrm{~ms}$ and the gradient pulses were optimized for each solvent ( $\delta / 2$ varied between 0.6 and $1.0 \mathrm{~ms}$ ). The results are reported in Table 2. To estimate the relative accuracy of these PFG MAS measurements, we also reported for each solvent (when available) the range of diffusion data obtained both by NMR and other methods, as given in reference [50], by considering the minimum and maximum $D$ values reported in the above mentioned reference. As can be seen in Table 2, a satisfactory agreement is achieved between the PFG MAS self-diffusion data and those commonly found in the literature.

On the other hand, we checked whether self-diffusion coefficients could be accurately measured for more slowly diffusing species. Table 2 shows that the results obtained by PFG MAS at $4 \mathrm{kHz}$ for DMSO, formamide and octanol, 

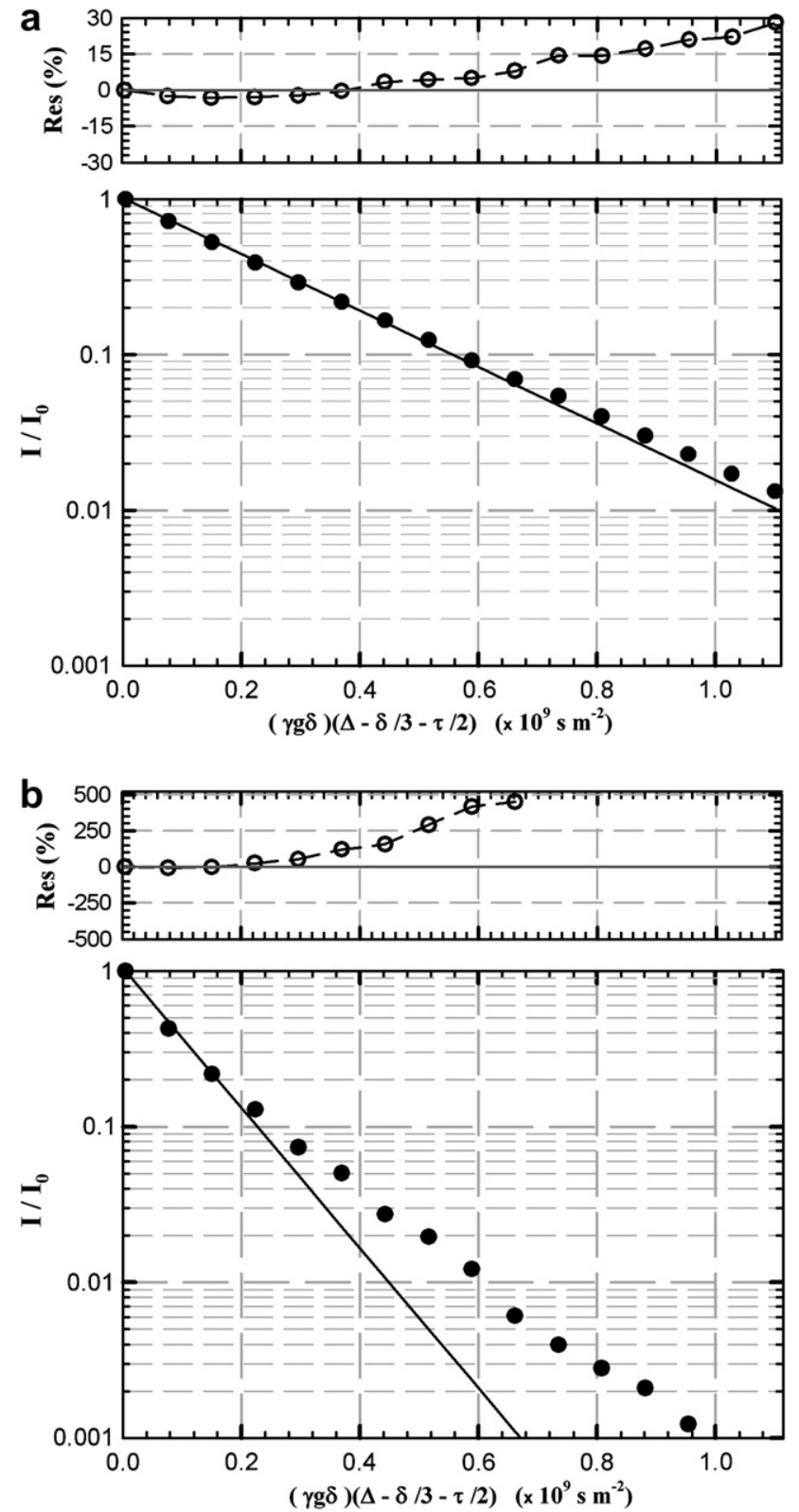

Fig. 4. PFG MAS signal decays obtained at $298 \mathrm{~K}$ on a HR MAS probe at $4 \mathrm{kHz}$ for acetonitrile with a (a) $12 \mu \mathrm{L}$ and (b) $50 \mu \mathrm{L}$ rotor. The diffusion time and gradient pulse duration were 80 and $0.6 \mathrm{~ms}$, respectively. The residuals are expressed as the difference between the experimental and adjusted data points, given in $\%$ with respect to the adjusted points.

were in good agreement with literature data. Note that these substances were chosen for illustrative purpose only.

To complete the range of investigated mobility, we analyzed a relatively large poly(ethylene oxide) sample (PEO, $M_{\mathrm{w}} 116 \mathrm{kDa}$ ) dissolved both in deuterated water and deuterated chloroform (about $1.5 \mathrm{~g} \mathrm{~L}^{-1}$ in both cases). In this latter case, the temperature was set to $300 \mathrm{~K}$ to compare the results with available literature data, and two spinning rates were chosen, 3 and $4 \mathrm{kHz}$ (according to Fig. 3).

The PEO self-diffusion coefficients in $\mathrm{D}_{2} \mathrm{O}$ and $\mathrm{CDCl}_{3}$ were $2.0 \pm 0.1 \times 10^{-11}$ and $3.5 \pm 0.1 \times 10^{-11} \mathrm{~m}^{2} \mathrm{~s}^{-1}$, respec-
Table 2

Self-diffusion coefficients $\left(10^{-9} \mathrm{~m}^{2} \mathrm{~s}^{-1}\right)$ obtained at $298 \mathrm{~K}$ for a few selected compounds on a HR MAS probe with a $12 \mu \mathrm{L}$ rotor and a spinning rate of $4 \mathrm{kHz}$

\begin{tabular}{llll}
\hline Compound & $D_{\mathrm{MAS}}$ & $D_{\mathrm{NMR}}{ }^{\mathrm{a}}$ & $D_{\text {other methods }}{ }^{\mathrm{a}}$ \\
\hline Acetone & 4.53 & $4.57^{\mathrm{b}}$ & - \\
Acetonitrile & 4.18 & $4.29-4.39$ & $4.34-5.4$ \\
Benzene & 2.18 & $2.1-2.26$ & $2.13-2.27$ \\
Chloroform & 2.45 & $2.33-2.83$ & 2.42 \\
Cyclohexane & 1.45 & $1.42-1.47$ & $1.43-1.47$ \\
Dichloromethane & 3.53 & $3.48-4.00$ & - \\
Ethanol & 0.96 & $1.0-1.08$ & $1.01-1.05$ \\
Hexane & 4.00 & $4.2-4.28$ & $4.13-4.26$ \\
Methanol & 2.26 & $2.3-3.50$ & $2.21-2.42$ \\
Water & 2.29 & $2.30^{\mathrm{c}}$ & - \\
Water (traces in $\left.\mathrm{D}_{2} \mathrm{O}\right)$ & 1.83 & $1.90^{\mathrm{c}}$ & - \\
Dimethylsulphoxide & 0.74 & $0.73^{\mathrm{c}}$ & - \\
Formamide & 0.51 & $0.55^{\mathrm{b}}$ & - \\
Octanol & 0.16 & $0.14^{\mathrm{d}}$ & - \\
\hline
\end{tabular}

Literature values are also reported.

${ }^{\text {a }}$ From Ref. [50].

${ }^{\mathrm{b}}$ From Ref. [62].

c From Ref. [55].

d From Ref. [63].

tively, which agreed with available data $\left(2.1 \times 10^{-11}\right.$ and $3.4 \times 10^{-11} \mathrm{~m}^{2} \mathrm{~s}^{-1}$ ) obtained by conventional liquid-state NMR diffusion experiments (references [61] and [64], respectively). While for $\mathrm{D}_{2} \mathrm{O}$ no significant change was observed for the measurements performed between 3 and $4 \mathrm{kHz}$, the increase in spinning rate (from 3 to $4 \mathrm{kHz}$ ) led for $\mathrm{CDCl}_{3}$ to an apparent increase in the self-diffusion coefficient (the latter value being about $40 \%$ larger). The corresponding PFG decays are shown in Fig. 5. Note that for $\mathrm{CDCl}_{3}$ at $3 \mathrm{kHz}$ (Fig. 5b), the first point was systematically an outliar and was hence removed prior to data fitting, which explains why only 15 data points are present (instead of 16). Overall, this figure shows that, even for slowly diffusing species, satisfactory linear PFG decays could be achieved.

\section{Conclusion}

We have shown that self-diffusion coefficients can be measured in PFG MAS diffusion studies of liquids, both for fast and slowly diffusing molecules, as long as the rotor active volume is kept to a minimum. Specifically, a $12 \mu \mathrm{L}$ rotor was shown here to be appropriate whereas a $50 \mu \mathrm{L}$ rotor was clearly inadequate and led to erratic estimations, especially for low viscous samples. Spinning rates can be conveniently set between 3 and $6 \mathrm{kHz}$, but should not be higher than 3 or $4 \mathrm{kHz}$ when analyzing slowly diffusing species with long diffusion times, especially when these species are dissolved in low viscous solvents such as $\mathrm{CDCl}_{3}$. Therefore, care must be taken when calibrating the gradient strength of a MAS probe by measuring the self-diffusion coefficient of a spinning substance of known diffusivity, since false measurements will lead to wrong estimates of 

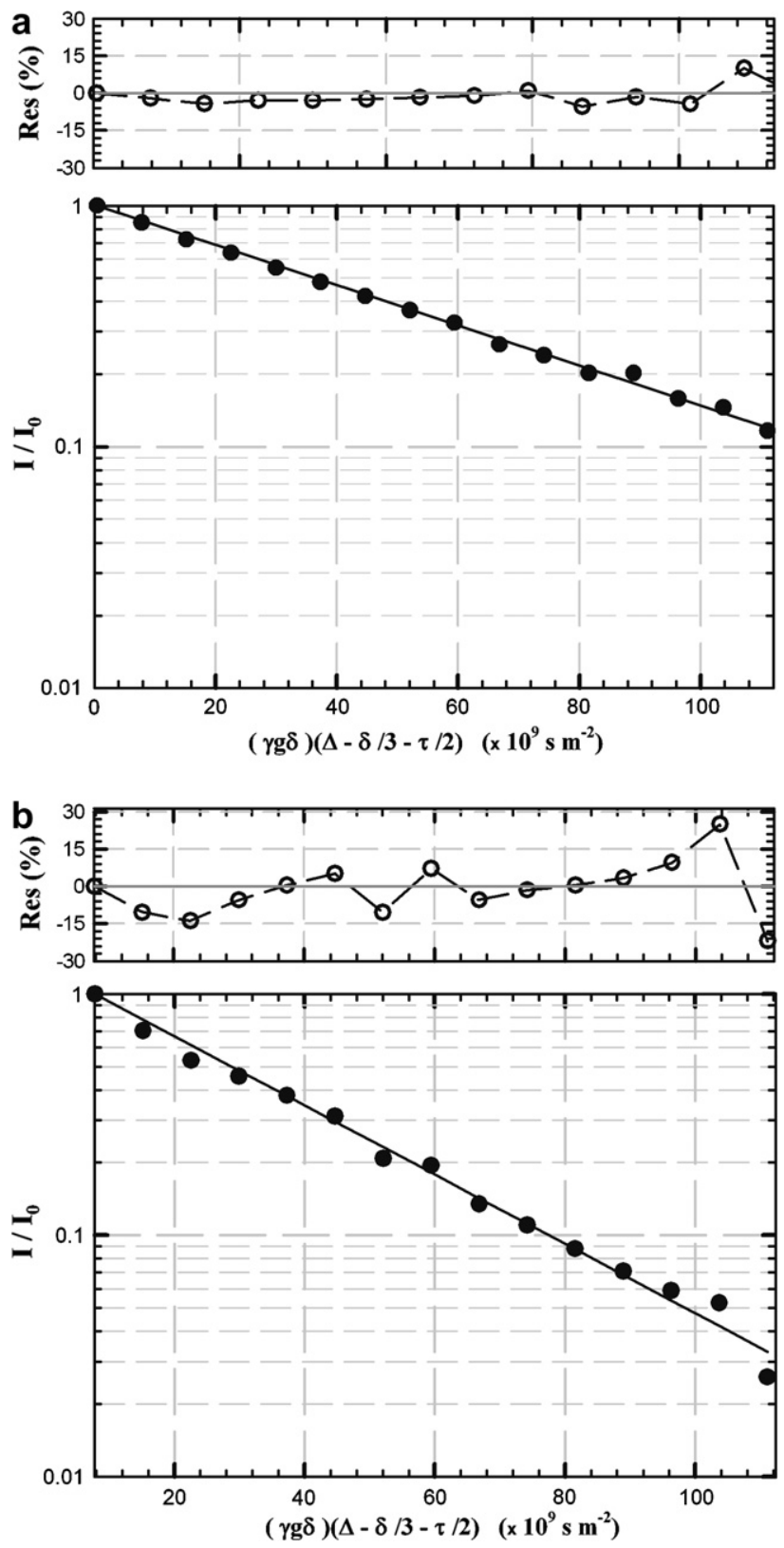

Fig. 5. PFG MAS signal decays obtained at $300 \mathrm{~K}$ for a PEO $\left(M_{\mathrm{w}}\right.$ $116 \mathrm{kDa}, 1.5 \mathrm{~g} \mathrm{~L}^{-1}$ ) sample on a HR MAS probe with a $12 \mu \mathrm{L}$ rotor in (a) $\mathrm{D}_{2} \mathrm{O}$ and (b) $\mathrm{CDCl}_{3}$, at 4 and $3 \mathrm{kHz}$, respectively. The diffusion time was $600 \mathrm{~ms}$ whereas the gradient pulse duration was 2.0 and $2.2 \mathrm{~ms}$, respectively.

the gradient coil constant. In addition, similarly to liquid state NMR, the accuracy achieved in this work might be further improved by using other pulse sequences. Note that no rotor synchronization was performed. Moreover, because the MAS probe offers an excellent gradient uniformity, MAS diffusion studies allow for an interesting alternative to conventional methods used for avoiding artefacts caused by gradient poor uniformity. Finally, in addition to providing a method to measure accurate selfdiffusion coefficients of sample-limited mixtures, another potential application of this work is the possibility to avoid radiation damping interferences in self-diffusion measurements on high field NMR instruments.

\section{Acknowledgments}

Thanks are due to the Spectropole for allowing special access to the NMR facilities of the Fédération des Sciences Chimiques de Marseille. The authors thank D. Cross from ROTOTEC-SPINTEC (Biebesheim, Germany) for providing them with the $4 \mathrm{~mm}$ rotor prototype mentioned in the text.

\section{References}

[1] P.T. Callaghan, Principles of Nuclear Magnetic Resonance Microscopy, Oxford University Press, New York, 1991.

[2] E.O. Stejskal, J.E. Tanner, Spin diffusion measurements: spin echoes in the presence of a time-dependent field gradient, J. Chem. Phys. 42 (1965) 288-291.

[3] K.F. Morris, C.S. Johnson Jr., Diffusion-ordered two-dimensional nuclear magnetic resonance spectroscopy, J. Am. Chem. Soc. 114 (1992) 3139-3141.

[4] P. Stilbs, Fourier transform pulsed-gradient spin-echo studies of molecular diffusion, Prog. Nucl. Magn. Reson. Spectrosc. 19 (1987) $1-45$.

[5] O. Söderman, P. Stilbs, NMR studies of complex surfactant systems, Prog. Nucl. Magn. Reson. Spectrosc. 26 (1994) 445-482.

[6] E.D. von Meerwall, Self-diffusion in polymer systems, measured with field-gradient spin echo NMR methods, Adv. Polym. Sci. 54 (1983) $1-29$.

[7] F. Stallmach, J. Kärger, The potentials of pulsed field gradient NMR for investigation of porous media, Adsorption 5 (1999) 117-133.

[8] A.R. Waldeck, P.W. Kuchel, A.J. Lennon, B.E. Chapman, NMR diffusion measurements to characterise membrane transport and solute binding, Prog. Nucl. Magn. Reson. Spectrosc. 30 (1997) $39-68$.

[9] T. Brand, E. Cabrita, S. Berger, Intermolecular interaction as investigated by NOE and diffusion studies, Prog. Nucl. Magn. Reson. Spectrosc. 46 (2005) 159-196.

[10] G. Lippens, M. Bourdonneau, C. Dhalluin, R. Warrass, T. Richert, C. Setharaman, C. Boutillon, M. Piotto, Study of compounds attached to solid supports using high resolution magic angle spinning NMR, Curr. Org. Chem. 3 (1999) 147-169.

[11] W.P. Power, High resolution magic angle spinning-applications to solid phase synthetic systems and other semi-solids, in: G. Webb, (Ed.), Annual Reports on NMR Spectroscopy, London, 2003, pp. 261-295.

[12] W.E. Maas, F.H. Laukien, D.G. Cory, Gradient, high resolution, magic angle sample spinning NMR, J. Am. Chem. Soc. 118 (1996) 13085-13086.

[13] C. Malveau, D. Grandclaude, P. Tekely, F. Beaume, D. Canet, Spatially resolved crystalline and amorphous components of polymeric materials by carbon- 13 chemical shift imaging performed with radio-frequency field gradients, Macromolecules 34 (2001) 6274-6280.

[14] J. Chin, B. Fell, S. Pochapsky, M.J. Shapiro, J.R. Wareing, 2D SECSY NMR for combinatorial chemistry. High-resolution MAS spectra for resin-bound molecules, J. Org. Chem. 63 (1998) 13091311.

[15] R. Warrass, J.-M. Wieruszeski, G. Lippens, Efficient suppression of solvent resonances in HR-MAS of resin-supported molecules, J. Am. Chem. Soc. 121 (1999) 3787-3788.

[16] J. Chin, A. Chen, M.J. Shapiro, Improved high-resolution diffusion filtered ${ }^{1}$ H MAS NMR, Magn. Reson. Chem. 38 (2000) 782-784.

[17] O.M. Rooney, J. Troke, J.K. Nicholson, J.L. Griffin, High-resolution diffusion and relaxation-edited magic angle spinning ${ }^{1} \mathrm{H}$ NMR 
spectroscopy of intact liver tissue, Magn. Reson. Med. 50 (2003) 925930.

[18] P. Weybright, K. Millis, N. Campbell, D.G. Cory, S. Singer, Gradient, high-resolution, magic angle spinning ${ }^{1} \mathrm{H}$ nuclear magnetic resonance spectroscopy of intact cells, Magn. Reson. Med. 39 (1998) $337-345$.

[19] J.L. Griffin, J. Troke, L.A. Walker, R.F. Shore, J.C. Lindon, J.K. Nicholson, The biochemical profile of rat testicular tissue as measured by magic angle spinning ${ }^{1} \mathrm{H}$ NMR spectroscopy, FEBS Lett. 486 (2000) 225-229.

[20] Y. Pérez, H. Lahrech, M.E. Cabanas, R. Barnadas, M. Sabés, C. Rémy, C. Arus, Measurement by nuclear magnetic resonance diffusion of the dimensions of the mobile lipid compartment in C6 cells, Cancer Res. 62 (2002) 5672-5677.

[21] H.C. Gaede, K. Gawrisch, Lateral diffusion rates of lipid, water, and a hydrophobic drug in a multilamellar liposome, Biophys. J. 85 (2003) $1734-1740$.

[22] O. Wattraint, C. Sarazin, Diffusion measurements of water, ubiquinone and lipid bilayer inside a cylindrical nanoporous support: a stimulated echo pulsed-field gradient MAS-NMR investigation, Biochim. Biophys. Acta, Biomembr. 1713 (2005) 65-72.

[23] H.C. Gaede, K. Gawrisch, Multi-dimensional pulsed field gradient magic angle spinning NMR experiments on membranes, Magn. Reson. Chem. 42 (2004) 115-122.

[24] A. Pampel, D. Michel, R. Reszka, Pulsed field gradient MAS-NMR studies of the mobility of carboplatin in cubic liquid-crystalline phases, Chem. Phys. Lett. 357 (2002) 131-136.

[25] L. Shintu, S. Caldarelli, Toward the determination of the geographical origin of emmental(er) cheese via high resolution MAS NMR: a preliminary investigation, J. Agric. Food Chem. 54 (2006) 4148-4154.

[26] W.E. Maas, A. Bielecki, M. Ziliox, F.H. Laukien, D.G. Cory, Magnetic field gradients in solid state magic angle spinning NMR, J. Magn. Reson. 141 (1999) 29-33.

[27] I. Fischbach, K. Thieme, A. Hoffmann, M. Hehn, I. Schnell, PFGassisted selection and suppression of ${ }^{1} \mathrm{H}$ NMR signals in the solid state under fast MAS, J. Magn. Reson. 165 (2003) 102-115.

[28] A. Pampel, K. Zick, H. Glauner, F. Engelke, Studying lateral diffusion in lipid bilayers by combining a magic angle spinning NMR probe with a microimaging gradient system, J. Am. Chem. Soc. 126 (2004) 9534-9535.

[29] A. Pampel, F. Engelke, P. Galvosas, C. Krause, F. Stallmach, D. Michel, J. Kärger, Selective multi-component diffusion measurement in zeolites by pulsed field gradient NMR, Micropor. Mesopor. Mat. 90 (2006) 271-277.

[30] S.A. Bradley, J. Paschal, P. Kulanthaivel, DOSY of sample-limited mixtures: Comparison of cold, nano and conventional probes, Magn. Reson. Chem. 43 (2005) 31-35.

[31] Y. Maguire, I.L. Chuang, S. Zhang, N. Gershenfeld, Ultra-smallsample molecular structure detection using microslot waveguide nuclear spin resonance, Proc. Natl. Acad. Sci. USA 104 (2007) 91989203.

[32] P. Broekaert, J. Jeener, G. Lippens, J.M. Wieruszeski, MAS as a tool for suppressing dipolar field effects in high-resolution liquid NMR, J. Magn. Reson. 145 (2000) 259-261.

[33] W.S. Price, M. Wälchli, NMR diffusion measurements of strong signals: the PGSE-Q-switch experiment, Magn. Res. Chem. 40 (2002) S128-S132.

[34] M.A. Connell, A.L. Davis, A.M. Kenwright, G.A. Morris, NMR measurements of diffusion in concentrated samples: avoiding problems with radiation damping, Anal. Bioanal. Chem. 378 (2004) 15681573.

[35] S. Viel, F. Ziarelli, S. Caldarelli, Enhanced diffusion-edited NMR spectroscopy of mixtures using chromatographic stationary phases, Proc. Natl. Acad. Sci. USA 100 (2003) 9696-9698.

[36] G. Pagès, C. Delaurent, S. Caldarelli, Investigation of the chromatographic process via pulsed-gradient spin-echo nuclear magnetic resonance. Role of the solvent composition in partitioning chromatography, Anal. Chem. 78 (2006) 561-566.
[37] G. Pagès, C. Delaurent, S. Caldarelli, Simplified analysis of mixtures of small molecules by chromatographic NMR spectroscopy, Angew. Chem. Int. Ed. 45 (2006) 5950-5953.

[38] F. Ziarelli, S. Viel, S. Sanchez, D. Cross, S. Caldarelli, Precision and sensitivity optimization of quantitative measurements in solid state NMR, J. Magn. Reson. 188 (2007) 260-266.

[39] D. Wu, A. Chen, C.S. Johnson Jr., An improved diffusion-ordered spectroscopy experiment incorporating bipolar-gradient pulses, J. Magn. Reson., Ser. A 115 (1995) 260-264.

[40] W.S. Price, Pulsed-field gradient nuclear magnetic resonance as a tool for studying translational diffusion: Part 2. Experimental aspects, Concept Magn. Reson. 10 (1998) 197-237.

[41] B. Antalek, Using pulsed gradient spin echo NMR for chemical mixture analysis: how to obtain optimum results, Concept Magn. Reson. 14 (2002) 225-258.

[42] Y. Cohen, L. Avram, L. Frish, Diffusion NMR spectroscopy in supramolecular and combinatorial chemistry: an old parameter-new insights, Angew. Chem. Int. Ed. 44 (2005) 520-554.

[43] P.S. Pregosin, P.G. Anil Kumar, I. Fernández, Pulsed gradient spinecho (PGSE) diffusion and 1H, 19F heteronuclear overhauser spectroscopy (HOESY) NMR methods in inorganic and organometallic chemistry: something old and something new, Chem. Rev. 105 (2005) 2977-2998.

[44] A.W. Nicholls, R.J. Mortishire-Smith, Temperature calibration of a high-resolution magic-angle spinning NMR probe for analysis of tissue samples, Magn. Reson. Chem. 39 (2001) 773-776.

[45] C. Ammann, P. Meier, A. Merbach, A simple multinuclear NMR thermometer, J. Magn. Reson. (1969) 46 (1982) 319-321.

[46] I.V. Polozov, K. Gawrisch, Domains in binary SOPC/POPE lipid mixtures studied by pulsed field gradient ${ }^{1} \mathrm{H}$ MAS NMR, Biophys. J. 87 (2004) 1741-1751.

[47] T. Mildner, H. Ernst, D. Freude, ${ }^{207} \mathrm{~Pb}$ NMR detection of spinninginduced temperature gradients in MAS rotors, Solid State Nucl. Magn. Reson. 5 (1996) 269-271.

[48] P. Damberg, J. Jarvet, A. Gräslund, Accurate measurement of translational diffusion coefficients: a practical method to account for non linear gradients, J. Magn. Reson. 148 (2001) 343-348.

[49] R.E. Hurd, A. Deese, M.O. Johnson, S. Sukumar, P.C.M. van Zijl, Impact of differential linearity in gradient-enhanced NMR, J. Magn. Reson., Ser. A 119 (1996) 285-288.

[50] H. Kato, T. Saito, M. Nabeshima, K. Shimada, S. Kinugasa, Assessment of diffusion coefficients of general solvents by PFGNMR: investigation of the sources error, J. Magn. Reson. 180 (2006) 266-273.

[51] B. Antalek, M.J. Hewitt, W. Windig, P.D. Yacobucci, T. Mourey, K. Le, The use of PGSE NMR and DECRA for determining polymer composition, Magn. Reson. Chem. 40 (2002) S60-S71.

[52] P.J. Bowyer, A.G. Swanson, G.A. Morris, Analyzing and correcting spectrometer temperature sensitivity, J. Magn. Reson. 152 (2001) 234-246.

[53] M.D. Pelta, G.A. Morris, M.J. Stchedroff, S.J. Hammond, A oneshot pulse sequence for high-resolution diffusion-ordered spectroscopy, Magn. Reson. Chem. 40 (2002) S147-S152.

[54] N.M. Loening, J. Keeler, Temperature accuracy and temperature gradients in solution-state NMR spectrometers, J. Magn. Reson. 159 (2002) 55-61.

[55] M. Holz, H. Weingärtner, Calibration in accurate spin-echo selfdiffusion measurements using ${ }^{1} \mathrm{H}$ and less-common nuclei, J. Magn. Reson. 92 (1991) 115-125.

[56] G.H. Sørland, D. Aksnes, Artefacts and pitfalls in diffusion measurements by NMR, Magn. Reson. Chem. 40 (2002) S139S146.

[57] R.L. Hurle, L.A. Woolf, Self-diffusion in liquid acetonitrile under pressure, J. Chem. Soc. Faraday Trans. 178 (1982) 2233-2238.

[58] M. Holz, S.R. Heil, A. Sacco, Temperature-dependent self-diffusion coefficients of water and six selected molecular liquids for calibration in accurate ${ }^{1} \mathrm{H}$ NMR PFG measurements, Phys. Chem. Chem. Phys. 2 (2000) 4740-4742. 
[59] P.T. Callaghan, M.E. Komlosh, M. Nyden, High magnetic field gradient PGSE NMR in the presence of a large polarizing field, J. Magn. Reson. 133 (1998) 177-182.

[60] F.D. Doty, G. Entzminger, Y.A. Yang, Magnetism in high-resolution NMR probe design. II: HR MAS, Concept Magn. Reson. 10 (1998) 239-260.

[61] M. Mazarin, S. Viel, B. Allard-Breton, A. Thevand, L. Charles, Use of pulsed gradient spin-echo NMR as a tool in MALDI method development for polymer molecular weight determination, Anal. Chem. 78 (2006) 2758-2764.

[62] M. Holz, X. Mao, D. Seiferling, A. Sacco, Experimental study of dynamic isotope effects in molecular liquids. Detection of translationrotation coupling, J. Chem. Phys. 104 (1996) 669-679.

[63] M. Holz, A. Sacco, Self-diffusion coefficients of various liquids at $25^{\circ} \mathrm{C}$, Almanach Bruker (2001) 72

[64] Unpublished results. 